\title{
Laser Acceleration of Protons from Thin Film Targets
}

\author{
K. Flippo, 'S. Banerjee, ${ }^{\dagger}$ V. Yu. Bychenkov, ${ }^{\dagger}$ S. Gu, ${ }^{\dagger}$ A. Maksimchuk, \\ G. Mourou, ${ }^{\dagger}$ K. Nemoto ${ }^{+}$and D. Umstadter ${ }^{\dagger}$ \\ ${ }^{+}$Center for Ultrafast Optical Science, University of Michigan, Ann Arbor, MI 48109-2099, USA \\ ${ }^{7} P$. N. Lebedev Physics Institute, Russian Academy of Science, Moscow 117924, Russia \\ ${ }^{+}$Central Research Institute of Electric Power Industry, 2-11-1, Iwado-kita, Komae-shi, \\ Tokyo, 201-8511 JAPAN
}

\begin{abstract}
A collimated beam of fast protons, with energies as high as $10 \mathrm{MeV}$ and total number of $10^{9}$, confined in a cone angle of $40^{\circ} \pm 10^{\circ}$ has been observed when a $10 \mathrm{TW}$ laser with frequencies either $\omega_{0}$ (corresponding to $1 \mu \mathrm{m}$ ) or $2 \omega_{0}$ was focused to an intensity of a few times $10^{18} \mathrm{~W} / \mathrm{cm}^{2}$ on the surface of a thin film target. The protons, which originate from impurities on the front side of the target, are accelerated over a region extending into the target and exit out the backside in a direction normal to the target surface. Acceleration field gradients of $\sim 10 \mathrm{GeV} / \mathrm{cm}$ are inferred. The maximum proton energy for $2 \omega_{0}$ can be explained by the charge-separation electrostatic-field acceleration due to "vacuum heating." In other set of experiments when a deuterated polystyrene layer was deposited on a surface of a Mylar film and $\mathrm{a}^{10} \mathrm{~B}$ sample was placed behind the target, we observed the production of $\sim 10^{5}$ atoms of positron active isotope ${ }^{11} \mathrm{C}$ from the nuclear reaction ${ }^{10} \mathrm{~B}(\mathrm{~d}, \mathrm{n}){ }^{11} \mathrm{C}$.
\end{abstract}

\section{INTRODUCTION}

The development of ultra-intense lasers using the Chirped Pulse Amplification technique has made intensities in excess of $10^{18} \mathrm{~W} / \mathrm{cm}^{2}$ routinely achievable. At these intensities an electron in a plasma, during a half laser cycle, can acquire energy that is equal to or greater then the electron's rest mass. Through the electrostatic field, caused by charge separation, the remaining ions can be accelerated to $\mathrm{MeV}$ energies. Since high-energy ions can participate in strong interactions, this opens a new realm dealing with the transformation of nuclei on a tabletop on a picosecond time scale.

Recently an interest has developed in ion acceleration by compact high-intensity subpicosecond lasers with potential applications for the initiation of nuclear reactions on a tabletop. Experiments now being carried out involve high-energy ions generated in the interaction of laser pulses with solid targets [1,2], gas jets [3,4], and clusters $[5,6]$. Most of the current research in this area is directed towards the development of a compact neutron source [7-10], while several other nuclear applications have recently been proposed: isotope production and initiation of fission reactions [11].

Critical for ion acceleration is the efficiency of laser-energy conversion into a highenergy electron component, since the latter through charge separation can produce the 
requisite strong electrostatic fields, $E$. Thermal expansion of a laser-driven plasma and ponderomotive electron expulsion constitute the most well-known examples of electrostatic-field production. While the former mechanism has been observed for many years [12], the latter one has only recently been observed in experiments with gas targets $[3,4]$.

In this paper, we report on the direct observation of a high-energy ion beam accelerated by an electrostatic field [13] in the forward (laser) direction to an energy as high as $\sim 10 \mathrm{MeV}$ with the laser intensity of $6.10^{18} \mathrm{~W} / \mathrm{cm}^{2}$, and the observation of nuclear fusion reactions induced by high-energy deuterons.

\section{ION ACCELERATION}

In our experiments we observed that the protons, which appear to originate from impurities on the front side of the thin-film target, are accelerated over a region extending into the target and exit out the back side, in a direction normal to the target surface. The results are found to depend on a plasma scale length and for a high lasercontrast case $\left(2 \omega_{0}\right) \mathrm{c}$ an be explained by the "vacuum heating" mechanism at a sharp interface due to the Brunel effect [14] or the $\mathbf{v} \times \mathbf{B}$ Lorentz force [15]. In previously reported experimental studies [1], the accelerated ions were found to propagate in the direction of plasma expansion, and the acceleration attributed to charge displacement by thermal expansion.

The experiments were performed using a $10 \mathrm{TW}$ hybrid Ti:Sapphire/Nd:phosphate glass chirped pulse amplification (CPA) laser, which is able to deliver up to $4 \mathrm{~J}$, in a $400 \mathrm{fs}$ pulse at the fundamental wavelength of $1.053 \mu \mathrm{m}$ with the intensity contrast ratio of $\sim 10^{4}: 1$, as measured by the third order correlation technique, and $1 \mathrm{~J}$ at the second harmonic with the contrast improved to an estimated $10^{7}: 1$ by the frequency doubling in a 4-mm KDP crystal. The experiments with the green light were limited to $1 \mathrm{~J}$, because of the nonlinear distortion of the laser wave front in the crystal. The laser beam was focused with an $\mathrm{f} / 3(\mathrm{f}=16.5 \mathrm{~cm})$ off-axis parabolic mirror on the surface of a thin foil targets. The maximum focused intensity was $6.10^{18} \mathrm{~W} / \mathrm{cm}^{2}$ for the fundamental frequency and $3.10^{18} \mathrm{~W} / \mathrm{cm}^{2}$ for $2 \omega_{0}$.

\section{Experiments at the Second Harmonic}

The $2 \omega_{0}$ laser light was focused on the surface of thin aluminum films with a thickness of $1.8 \mu \mathrm{m}$ at $0^{\circ}$ or $45^{\circ}$ incidence angle with an off-axis parabolic mirror to $3.10^{18} \mathrm{~W} / \mathrm{cm}^{2}$.

The high-energy ion emissions were recorded by CR-39 plastic nuclear track detectors, which are able to record ions with energies $\geq 100 \mathrm{keV} /$ nucleon as tracks on the surface of the detector, after being etched in sodium hydroxide solution.

To study the angular ion distribution we have used CR-39 covered with single thickness Mylar filters ranging from 2 to $26 \mu \mathrm{m}$. To determine the maximum energy and spectrum of the ions, the detectors were covered with steps of Mylar filters with thicknesses from 2 to $50 \mu \mathrm{m}$. To compare ion emission in the forward (through the 
thin-film target) and backward directions (the direction of plasma expansion) for $45^{\circ}$ incidence angle, two CR-39 detectors, with sets of Mylar filters, were placed both in front and in back of the target.

It was found that the predominant component of the high-energy ion emission is protons. These protons originate from a thin layer on the target surface contaminated with hydrocarbon and/or water vapor. Such target contamination was observed in the late 1960 s in laser-matter interaction experiments with nanosecond laser pulses as discussed in Ref. [12] and has been proven in the 1990s in experiments with subpicosecond high-intensity pulses [1,2]. In our experiments, we observed a highenergy proton beam, propagating inside a thin-film target and emerging through the rear surface as a beam with the angular divergence of about $40^{\circ} \pm 10^{\circ}$ (Fig. 1a). The proton angular distribution is peaked in the center of the beam, where the maximum density of ion tracks was observed (Fig. 1a). The direction of the high-energy proton beam does not depend on the angle of incidence of laser radiation and is always normal to the target surface. From this fact one would conclude that the electric field

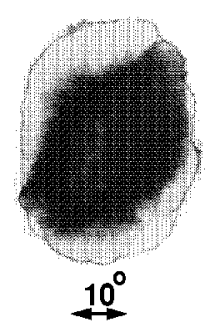

(a)

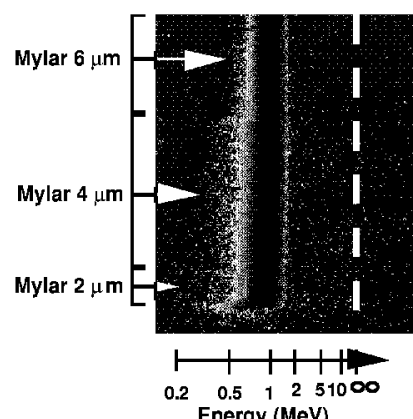

(b)

Figure 1. (a) A typical image of a proton beam observed in the forward (behind the target) direction in the interaction of $1.8-\mu \mathrm{m}$ thick aluminum foil with a high-intensity laser. The proton beam passed through a $25 \mu \mathrm{m}$ Mylar filter, which corresponds to energy above $1.2 \mathrm{MeV}$. The laser intensity on target is $2.10^{18} \mathrm{~W} / \mathrm{cm}^{2}$ at $2 \omega_{0}$ and 0 degree angle of incidence; (b) Spectrogram of fast protons emitted in the forward direction and deflected in a dipole magnetic spectrometer. A dashed line shows the position of the slit image without the magnet. CR-39 was used as a detector and covered with three steps of Mylar filter of 2, 4 and 6- $\mu \mathrm{m}$ thickness, which corresponds to proton cut-off energies of $0.2,0.3$ and $0.5 \mathrm{MeV}$.

of the charge separation, accelerating protons inside the target, is directed along the target surface normal.

To verify that most of the energetic ions are indeed protons, we used a dipole magnetic spectrometer with a $200-\mathrm{m} \mathrm{m}$ slit in front of the magnet. CR-39 plastic with three steps of Mylar film in front was used as a detector. The Mylar filters had thicknesses of 2,4 , and $6 \mu \mathrm{m}$, which correspond to proton cutoff energies of $0.2,0.3$, and $0.5 \mathrm{MeV}$, respectively. Figure $1 \mathrm{~b}$ presents a spectrogram of high-energy ions obtained with the dipole magnet. It shows a very sharp cutoff for particle energies of about $1.5 \mathrm{MeV}$, which was consistent with results on maximum proton energies as will 
be discussed below. On the left side of the spectrogram (Fig. 1b) by the arrows we have shown positions of above-mentioned cutoff energies.

These positions agree within $\pm 10 \%$ of accuracy to the deflection of protons in the magnetic field, expressed by the formula: $x \approx L Z e b B / \sqrt{2 \varepsilon_{i} M}$, where $L$ is the distance between magnet and detector, $Z=1$ for protons, $e$ is the electron charge, $b$ is the width of the magnet, $B$ is the magnetic field strength, and $\varepsilon_{i}$ and $M$ are the ion energy and mass.

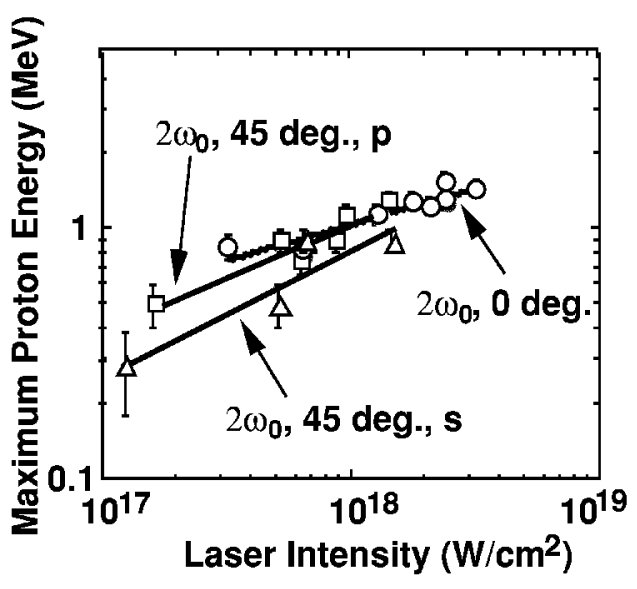

(a)

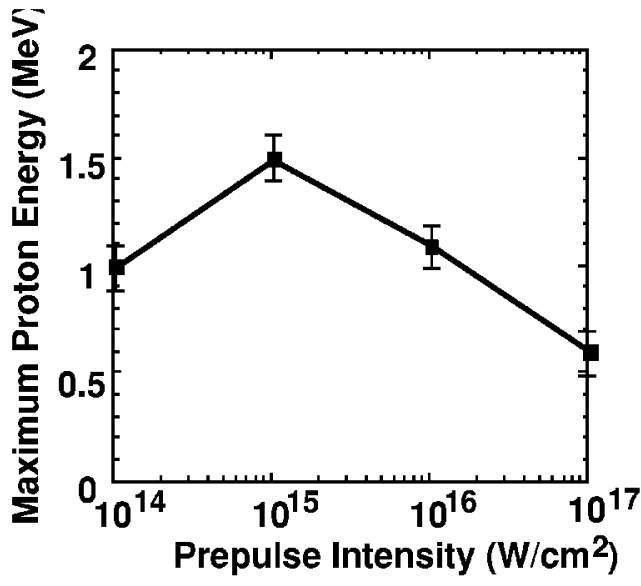

(b)

Figure 2. The maximum proton energy in the forward direction as a function of (a) laser intensity at $2 \omega_{0}$ and for different conditions of illumination of $1.8-\mu \mathrm{m} \mathrm{Al}$ foil: circles $-0^{\circ}$; squares $-45^{\circ}, p-$ polarization; triangles $-45^{\circ}, s$ - polarization. Solid lines are the best power function fits to the experimental data. (b) The $2 \omega_{0}$ prepulse intensity at $10^{18} \mathrm{~W} / \mathrm{cm} 2$ main pulse intensity.

We studied the dependence of the maximum proton energy versus the laser intensity for different conditions of $\mathrm{Al}$ thin foil illumination. The highest proton energy of $1.6 \mathrm{MeV}$ was observed for normal incidence at the maximum laser intensity of $3.10^{18} \mathrm{~W} / \mathrm{cm}^{2}$ (Fig. 2a). For $45^{\circ}$ angle of incidence the highest intensity on a target was reduced by a factor of 2 due to increased spot size in the horizontal direction. Maximum observed proton energy, $\varepsilon_{i}{ }^{\max }$, was comparable for both cases for the same intensity and can be fitted as a function $\varepsilon_{i}{ }^{\max } \propto I^{\alpha}$, where $\alpha$ is between 0.3 and 0.4 . Illumination with $s$ polarization at $45^{\circ}$ has produced protons with energies of 200-300 $\mathrm{keV}$ less than for $p$ polarization. We also observed high-energy protons in the backward direction (in the direction of a plasma expansion) for $45^{\circ}$ laser illumination, but their energy was twice lower than those in the forward direction. We changed the plasma gradient scale length by introducing a prepulse at $2 \omega_{0}$, with the time delay of $50 \mathrm{ps}$ in front of the main $2 \omega_{0}$ pulse. We varied the prepulse intensity from $0.01 \%$ to $10 \%$ of main pulse intensity of $10^{18} \mathrm{~W} / \mathrm{cm}^{2}$. We found that there is an optimum in prepulse intensity of about $10^{15} \mathrm{~W} / \mathrm{cm}^{2}$ (Fig. 2b) for maximum proton energy production. 
We estimated that at this intensity the scale length of the preformed plasma is about a few laser wavelength. The proton energy spectrum was measured by the foil attenuation method. Using steps of Mylar filters, with thicknesses differing by $2 \mu \mathrm{m}$ provided proton energy resolution of about $100 \mathrm{keV}$. The proton spectrum (Fig. 3a) shows an exponential decay from energies of $400 \mathrm{keV}$ to $1 \mathrm{MeV}$ with a characteristic temperature of $230 \mathrm{keV}$ (solid line). An interesting feature of the spectrum at higher energy is a plateau, which ends in a sharp energy cutoff at $1.5 \mathrm{MeV}$ that is typical for the electrostatic mechanism of ion acceleration [11].

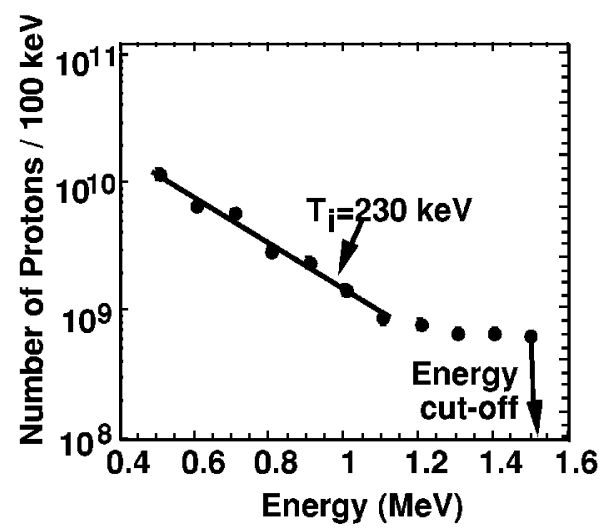

(a)

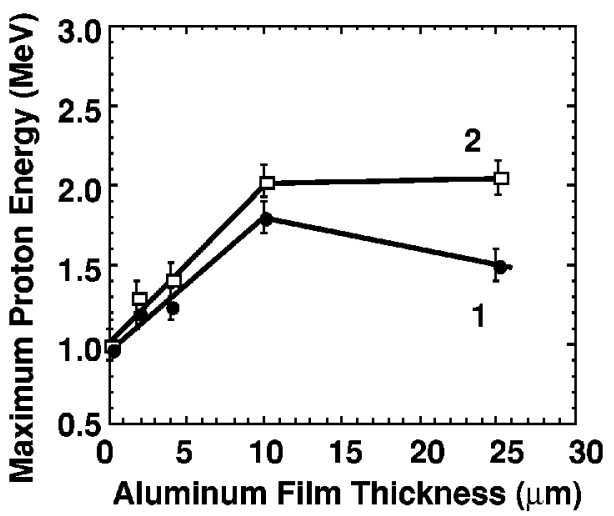

(b)

Figure 3. (a) Energy distribution of fast protons in the forward direction (circles), measured by attenuation of the beam in Mylar filters of different thicknesses for $2 \omega_{0}$ illumination of 1.8- $\mu \mathrm{m} \mathrm{Al} \mathrm{film}$ with intensity of $2 \times 10^{18} \mathrm{~W} / \mathrm{cm}^{2}$ at normal incidence. An arrow shows a sharp cutoff in proton energy on a spectral distribution below a detection threshold. The detection threshold was about $10^{6}$ protons_100 $\mathrm{keV}$. (b) Maximum energy of protons for laser intensity of $1.5 \times 10^{18} \mathrm{~W} / \mathrm{cm}^{2}$ at $2 \omega_{\mathrm{n}}$ and $0^{\circ}$ angle of incidence as a function of aluminum foil thickness - experiment (squares); ion losses are excluded (circles).

To determine the scale length of ion acceleration we varied the thickness of aluminum film from 0.1 to $25 \mu \mathrm{m}$. Circles on Fig. $3 \mathrm{~b}$ are the experimental data points showing the dependence of maximum proton energy versus thickness $l_{0}$ of the film. For the thinnest target with $l_{0}=0.1 \mu \mathrm{m}, \varepsilon_{i}{ }^{\max }$ was about $1 \mathrm{MeV}$. We observed an increase in the maximum detected proton energy versus target thickness for $l_{0}<10 \mu \mathrm{m}$. It reaches a peak at $l_{0} \approx 10 \mu \mathrm{m}$. At larger film thicknesses the proton energy decreases. This experiment demonstrates very efficient ion acceleration at a short spatial scale at the target front side where the predominant ion acceleration occurs and where ions gain their characteristic energy. For the maximum laser intensity we also have studied the dependence of maximum proton energy as a function of atomic number $Z$ for $2 \mu \mathrm{m}$ thick carbon, Mylar, molybdenum, and lead foils. We did not observe a significant difference in the maximum proton energies for different materials.

In spite of the absence of self-consistent theory of high-energy electron and ion generation in the laser-solid tar-get interactions, we may propose a heuristic pragmatic 
approach to get qualitative estimations. We will consider an interaction of a highcontrast laser pulse with an intensity $I>10^{18} \mathrm{~W} / \mathrm{cm}^{2}$ at normal incidence in which the high-energy electrons with relativistic velocities $v \approx c$ can be produced. We assume when a high-intensity high-contrast laser pulse terminates at a target surface it produces a plasma with a size $l \approx \lambda / 2$ [16], due to the longitudinal electron oscillations resulting from $\mathbf{v} \times \mathbf{B}$ oscillating Lorentz force. Near the target-vacuum surface the electrons are pushed in and out by the oscillating component of the ponderomotive force. Inside the target this force sharply vanishes. Twice in a laser period electrons reenter the target. Returning electrons are accelerated by the "vacuum" electric field and then deposit their energy inside the target. The electrons of this plasma are strongly heated by the laser light, penetrate inside the solid target with relativistic velocities, and constitute a low density $\left(n_{e}<n_{c}\right)$ high-energy component of the entire electron population. They acquire an energy $\varepsilon_{e}$ from the laser pulse which can be evaluated from energy balance: $n_{e} \varepsilon_{e} c \approx \eta I$, where $n_{e}$ is the electron density of small size preformed plasma and coefficient $\eta$ defines the efficiency of laser-energy conversion into the high-energy electrons. For laser intensities in the range of $10^{17}$ $3.10^{18} \mathrm{~W} / \mathrm{cm}^{2}$ we estimate $\eta$ in a range from a few percent to $15 \%$, in accordance with previous measurements [17] in similar experimental conditions.

The mean free path of high-energy electrons is very large- $1 \mathrm{MeV}$ free electron penetrates solid aluminum over a distance of $\sim 1.5 \mu \mathrm{m}$. Nevertheless, in a plasma it is limited to a Debye length $\lambda_{D e}$ due to the charge-separation field, which for an electron density $n_{e} \sim 10^{19}-10^{20} \mathrm{~cm}^{-3}$ is comparable with the laser wavelength. High-energy electrons penetrating into a solid target (or even passing through it for the very thin foils) create an electrostatic field with $e \Phi \approx \varepsilon_{e}$, or $E \sim \varepsilon_{e} / \lambda_{D e} \sim 10 \mathrm{GeV} / \mathrm{cm}$, which accelerates ions in the forward direction, and they in turn decelerate. An electrostatic field near the target surface has a bipolar structure with the more pronounced component accelerating ions in a forward direction. If the laser pulse duration is larger than the ion acceleration time in the layer of size $\lambda_{D e}$, as is the case of our experiment, ions acquire an electrostatic energy $\varepsilon_{i} \approx Z e \Phi \approx Z \varepsilon_{e}$.

It has been known that the nonlinear regime of vacuum heating provides accelerated electrons with energies higher than predicted by the $\mathbf{v} \times \mathbf{B}$ force or $P$ component of the laser field. This is related to the strong "heating" due to the self-intersection of electron orbits [18]. Instead of returning to the target in each cycle, many electrons remain on the front of a target and form a time-averaged density profile. In order to blow the electrons from the preformed plasma layer, the laser intensity must be enough to accelerate electrons up to an energy exceeding the Coulomb energy [19]: $\varepsilon_{e} \geq 2$ $\pi e^{2} n_{e} l R$, where $R$ is the radius of focal spot, $R \gg l(R \cong 5 \mu \mathrm{m})$. From this relation and energy balance, one can estimate the characteristic electron density $n_{e} \leq n_{c}$

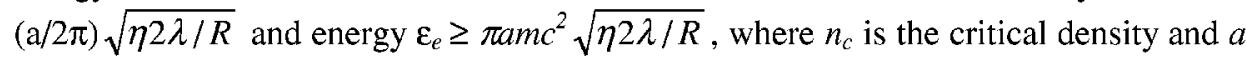
$=0.85 \times 10^{-9} \lambda[\mu \mathrm{m}] \sqrt{\left[\left[\mathrm{W} / \mathrm{cm}^{2}\right]\right.}$ is the normalized amplitude of laser-field vector potential. Consequently, the maximum ion energy can be evaluated as $\varepsilon_{i}^{\max } \geq$ $\mathrm{Z} \sqrt{\eta I R \lambda}$, where the laser intensity and spatial scales are measured in units of $10^{18}$ $\mathrm{W} / \mathrm{cm}^{2}$ and microns, respectively. For laser intensity of $3.10^{18} \mathrm{~W} / \mathrm{cm}^{2}$ and for $\eta=$ 
$10 \%, \varepsilon_{i}^{\max } \geq 1 \mathrm{MeV}$, that is, in agreement with the experimental data (Fig. 2a). We may compare our estimate with an estimate based on the ponderomotive potential [20], which has been used for the description of ion acceleration in gas targets $[3,11] \varepsilon_{i} \cong$ $Z m c^{2}\left[\sqrt{1+a^{2}}-1\right]$. This formula predicts an ion energy an order of magnitude lower than observed in the present experiment at an intensity of $3.10^{18} \mathrm{~W} / \mathrm{cm}^{2}$. It is evident also that the thermal expansion of a skin-layer plasma cannot explain $\mathrm{MeV}$ ion generation because of low bulk electron temperature there. One may believe that stochastic electron heating due to parametric processes in the plasma corona can provide the high temperature component of electrons, $T_{h}$, and corresponding high thermal expansion velocity $\sim \sqrt{T_{h} / M}$, where $M$ is the ion mass. However, at $2 \omega_{0}$ illumination with a high laser-intensity contrast the micron spatial scale of a plasma corona prevents such processes.

We may explain also why ion energy cutoff depends on the foil thickness (Fig. 3b, curve 1). As follows from the estimations, the typical $\lambda_{D e}$ is about $1 \mu \mathrm{m}$ so that for the foil thickness $l_{0} \leq 1 \mu \mathrm{m}$ electrons will penetrate to the backside. Even for thicker targets ultrafast electrons $(1 \mathrm{MeV})$, which cannot be stopped by charge separation, leave the target. Nevertheless, for the thick foils all electrons stop in the target and participate in generation of the electric field accelerating the ions. Because of this, one may expect an increase in the maximum ion energy with the foil thickness unless saturation occurs. However, ion energy losses increase with the foil thickness that should result in the optimum thickness, which according to Fig. 3b, curve 1, corresponds to $l_{0} \sim 10 \mu \mathrm{m}$. To demonstrate the effect of the saturation mentioned we plot also the similar dependence, $\varepsilon_{i}^{\max }\left(l_{0}\right)$ where ion energy losses are excluded (Fig. 3 b, curve 2).

\section{Experiments at the Fundamental Frequency}

In this section, we study the dependence of maximum proton energy as a function of laser intensity for the $\omega_{0}$ illumination (Fig. 4). For $\lambda=1.053 \mu \mathrm{m}$ the maximum proton energy was found to vary linearly with the laser intensity and reached $\sim 10 \pm 1$ $\mathrm{MeV}$ at $6.10^{18} \mathrm{~W} / \mathrm{cm}^{2}$.

Proton energy if given by the pondermotive potential of standing electromagnetic wave [12] is only $0.7 \mathrm{MeV}$, which is much less than the observed maximum proton energy. An estimation of the proton energy from the mechanism of "vacuum heating" [5] is closer to the observed value but also underestimates it by a few times. However, for the intensity contrast of $5.10^{5}: 1$ it is questionable to apply the latter estimation because a preplasma very likely appears before the maximum laser energy reaches the target. Thus, one may attribute such a preplasma as a reason for enhanced electron generation and, hence, enhanced electrostatic field, which efficiently accelerates the ions. Electrons accelerated in an underdense preplasma (electron density $n_{e}$ less than critical) up to the energy $\varepsilon^{m a x}$ can penetrate inside a solid target to their Debye length, $\lambda_{D e} \propto \sqrt{\varepsilon_{e} / n_{e}}$, and accelerate ions forward from the skin layer. Since the skin depth is shorter than $\lambda_{D e}$ a significant number of ions should have an energy equal to the 
electrostatic potential and the ion distribution function should demonstrate a plateau effect ("water bag" distribution) until energy cutoff $Z \varepsilon^{\max }$. Such an ion distribution function has already been observed in previous experiments [13].

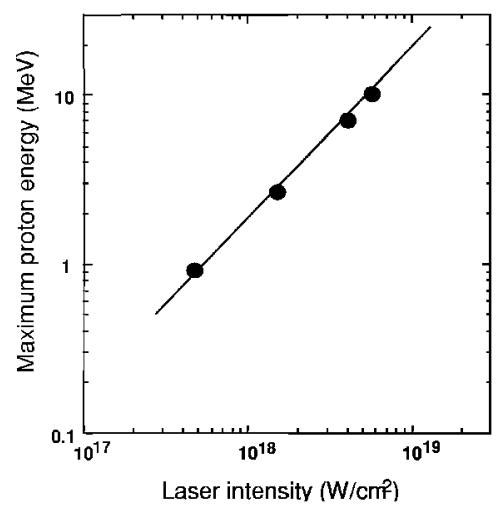

(a)

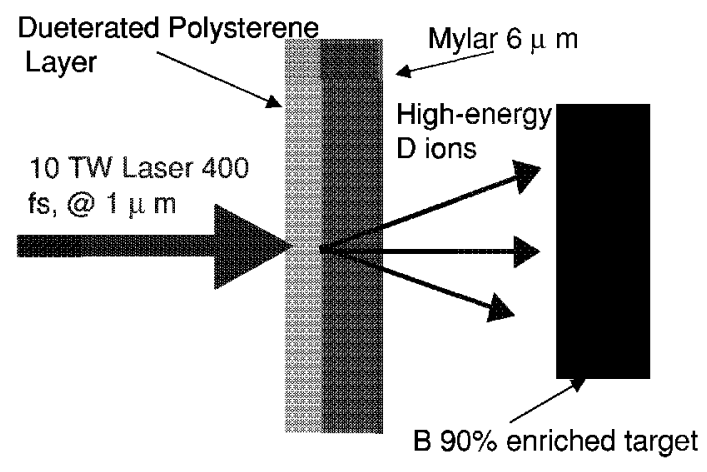

(b)

Figure. 4. (a) The maximum proton energy in the forward direction as a function of laser intensity $\omega_{n}$, $45^{0}$, p-polarization; (b) Schema for ${ }^{10} \mathrm{~B}$ activation with high-energy deuterons in the reaction ${ }^{10} \mathrm{~B}(\mathrm{~d}, \mathrm{n}){ }^{11} \mathrm{C}$

\section{NUCLEAR ACTIVATION}

After demonstrating acceleration of a significant amount of ions to high-energies in the forward direction we performed an experiment on nuclear activation [21]. We deposited a thin layer of a deuterated plastic on a front side of a $6 \mu \mathrm{m}$ thick Mylar foil. A $90 \%{ }^{10} \mathrm{~B}$ sample was positioned behind a thin film target at a distance of about $1 \mathrm{~cm}$ (Fig. 4b). A thin film target interacted with $1.053 \mu \mathrm{m}$ light at the intensity of $6.10^{18}$ $\mathrm{W} / \mathrm{cm}^{2}$. We expected to produce a positron active isotope ${ }^{11} \mathrm{C}$, which is a product of the reaction ${ }^{10} \mathrm{~B}(\mathrm{~d}, \mathrm{n}){ }^{11} \mathrm{C}$. A positron active isotope ${ }^{11} \mathrm{C}$ normally decays with the production of 2 photons with the energies of $511 \mathrm{keV}$ each, propagating in an opposite direction. We have used a standard coincidence event system, which consist of two channels with $\mathrm{NaI}$ scintillators coupled to a photomultiplier tubes (PMT), amplifiers and a timing unit. The two channels send signals to a fast coincidence unit with a timing window of $50 \mathrm{~ns}$ followed by a counter unit. This allowed us to detect simultaneously produced photons with an efficiency measured to be in the range of $0.05-0.1$. This was tested using a ${ }^{22} \mathrm{Na}$ radioactive source with a precisely known positron activity. The background noise was found to be $1-5 \mathrm{count} / \mathrm{min}$ and the radioactivity detection limit was $\sim 10 \mathrm{pCi}$.

After activating the ${ }^{10} \mathrm{~B}$ with the high-energy deuterons in a single shot we measured the radioactivity of produced ${ }^{11} \mathrm{C}$. Fig. 5a shows this result. On the same graph we plotted a theoretical decay for positron active isotope ${ }^{11} \mathrm{C}$ with a half-life of 20 minutes. These results indicates that we produced $\sim 10^{5}$ atoms of ${ }^{11} \mathrm{C}$, which corresponds to a radioactivity of $\sim 2 \mathrm{nCi}$ directly after the initial activation. Another interesting feature of this study is the very strong power dependence of ${ }^{11} \mathrm{C}$ yield as a 
function of the laser intensity. By changing the laser intensity factor of 2 (from $3.10^{18}$ $\mathrm{W} / \mathrm{cm}^{2}$ to $6.10^{18} \mathrm{~W} / \mathrm{cm}^{2}$ ) we have increased ${ }^{11} \mathrm{C}$ yield by almost 2 orders of magnitude (Fig. 5b).

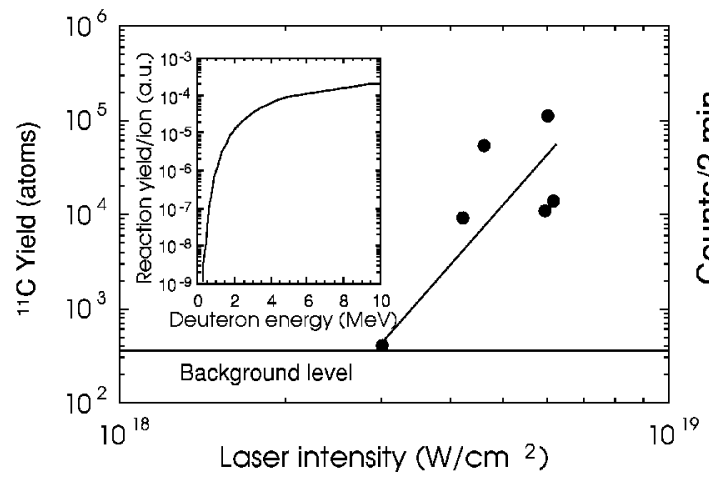

(a)

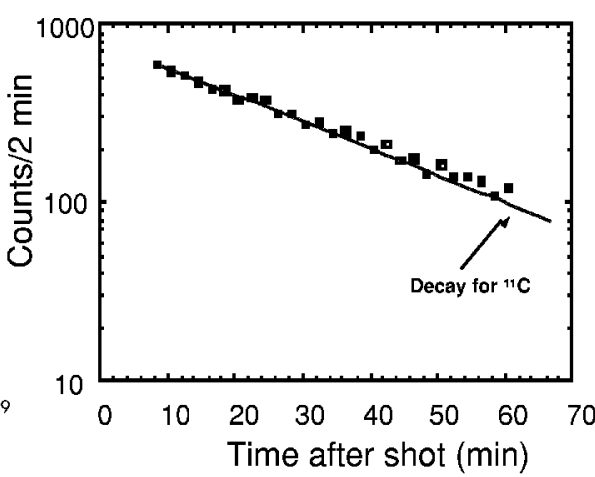

(b)

Figure 5. (a) Measured decay of radioactivity versus time for the reaction product ${ }^{10} \mathrm{~B}(\mathrm{~d}, \mathrm{n}){ }^{11} \mathrm{C}$ (squares). Solid line is a theoretical decay of ${ }^{11} \mathrm{C}$ with a half-life of 20 minutes; (b) ${ }^{11} \mathrm{C}$ yield versus laser intensity for $\omega_{0}$;

To verify that high-energy ions are accelerated from the front side of the target, that agrees with Ref. [22] and contradicts to Ref. [23], we irradiated a $6 \mu \mathrm{m}$ thick Mylar target with a layer of a deuterated plastic on its back side and performed the same ${ }^{10} \mathrm{~B}$ activation experiment described above [21]. In this case the activation signal was not above the background level. These results unequivocally indicate, that for the conditions of our experiment light ions are accelerated from the front side of the foil.

High-energy protons are also expected in the experiment with the layered deuterated target. They may participate in the ${ }^{11} \mathrm{~B}(\mathrm{p}, \mathrm{n}){ }^{11} \mathrm{C}$ reaction. The cross section of this reaction [24] has a higher threshold (above $3 \mathrm{MeV}$ ). Taking into account that the amount of ${ }^{11} \mathrm{~B}$ in boron sample was only $10 \%$, we expect ${ }^{11} \mathrm{C}$ yield for this reaction to be much less than that for deuterons. To verify that $(p, n)$ reaction does not contribute sufficiently in ${ }^{11} \mathrm{C}$ yield we focused the laser at its highest intensity on a bare Mylar film behind which was a boron sample. No activation signal was observed.

\section{SUMMARY}

In summary we have demonstrated the production of a directed beam of highenergy protons in the interaction of high-intensity laser with a thin-film target. These ions are accelerated by the electrostatic field of charge separation due to hot-electron electrons generated on a target front surface and driven inside the target by the laser. A maximum proton energy of about $1 \mathrm{MeV}$ has been observed for $2 \omega_{0}$ illumination and scales with the laser intensity as $\varepsilon_{i}^{\max } \sim I^{0.3}$. The maximum proton energy is about $10 \mathrm{MeV}$ and approximately proportional to the laser intensity, $I$, for the fundamental. And in both cases the proton energy is many more times higher than estimated from 
the pondermotive potential. We have also demonstrated the production of $\sim 10^{5}$ atoms of a positron active isotope ${ }^{11} \mathrm{C}$ from the ${ }^{10} \mathrm{~B}(\mathrm{~d}, \mathrm{n}){ }^{11} \mathrm{C}$ reaction. The activation results have shown that the generation of fast ions occurs on the front side of the target and the maximum proton energy agrees with an estimation of charge separation due to hotelectron generation by the vacuum heating effect for the $2 \omega_{0}$ case. A beam of energetic protons might one day serve as a high-current proton injector or be used in basic science to study nuclear transformations on a picosecond time scale. A charge compensated high-energy deuteron beam can also be used as a fast ion ignitor for the direct and indirect drive inertial confinement fusion research.

\section{ACKNOWLEDGMENTS}

The High Energy Physics Division of the U.S. Department of Energy supported the Michigan participants and the National Science Foundation supported the laser facility. The work of V. Yu. B. was supported by the Russian Foundation for Basic. S. B. is thankful for the support of CUOS Fellowship Program and K. N. would like to thank S. Akita, S. Sasaki and T. Suzuki for general support.

\section{REFERENCES}

1. P. Fews et al., Phys. Rev. Lett. 73, 1801 (1994); F. N. Beg et al., Phys. Plasmas 4, 447 (1997).

2. P. E. Young et al., Phys. Rev. Lett. 76, 3128 (1996).

3. G. S. Sarkisov et al., JETP Lett. 66, 828 (1997); G. S. Sarkisov et al., Phys. Rev. E 59, 7042 (1999).

4. K. Krushelnick et al., Phys. Rev. Lett. 83, 737 (1999).

5. T. Ditmire et al., Nature (London) 386, 54 (1997).

6. M. Lezius et al., Phys. Rev. Lett. 80, 261 (1998).

7. P. A. Norreys et al., Plasma Phys. Controlled Fusion 40, 175 (1998).

8. G. Pretzler et al., Phys. Rev. E 58, 1165 (1998).

9. T. Ditmire et al., Nature (London) 398, 489 (1999).

10. L. Disdier et al., Phys. Rev. Lett. 82, 1454 (1999).

11. V. Yu. Bychenkov et al., Sov. Phys. JETP 88, 1137 (1999).

12. S. J. Gitomer et al., Phys. Fluids 29, 2679 (1986).

13. A. Maksimchuk, et al., Phys. Rev. Lett. 84, 4108 (2000)

14. F. Brunel, Phys. Rev. Lett. 59, 52 (1987).

15. W. L. Kruer and K. Estabrook, Phys. Fluids 28, 430 (1985).

16. W. Yu et al., Phys. Rev. E 58, 2456 (1998).

17. J. Yu et al., Phys. Plasmas 6, 1318 (1999).

18. S. V. Bulanov et al., Phys. Plasmas 1, 745 (1994).

19. T. Zh. Esirkerov et al., JETP Lett. 70, 82 (1999).

20. S. C. Wilks, Phys. Fluids B 5, 2603 (1993).

21. K. Nemoto et al. (to be published in $A P L$ )

22. E.L.Clark, et al., Phys. Rev. Lett. 84, 670 (2000)

23. S.P. Hatchett, et al., Phys. Plasmas 7, 2076 (2000)

24. Experimental Nuclear Reaction Data File, http://www.nndc.bnl.gov/nndc/exfor 